\title{
Laparoscopic Repair of an Abdominal Intercostal Hernia Following Blunt Trauma: A Case Report
}

\author{
Benjamin Rebein ${ }^{a}$, Elias Fakhourya, b, Daria Abolghasemi ${ }^{a}$, Hoan Bui ${ }^{\mathrm{a}}$
}

\begin{abstract}
An abdominal intercostal hernia under an intact diaphragm is a rare phenomenon in which abdominal contents enter the intercostal space directly from the peritoneum through an acquired defect. The management of such cases has not been well established due to its infrequency. We discuss the successful laparoscopic management of such a hernia.
\end{abstract}

Keywords: Intercostal hernia; Hernia; Laparoscopic; Diaphragm

\section{Introduction}

Abdominal intercostal hernias are rare and an abdominal intercostal hernia under an intact diaphragm is an even more infrequent event. As such, the repair of such hernias is not well documented, with the majority of available literature describing the use of open techniques for repair. This is a case report describing the immediate successful laparoscopic repair of a traumatic abdominal intercostal hernia with an intact diaphragm in a 76-year-old Caucasian male. Our aim was to demonstrate that laparoscopic repair of this injury may be a viable option in patients without other major injuries or intraabdominal contamination.

\section{Case Report}

A 76-year-old male presented to the emergency department for evaluation by the trauma team immediately following a fall at a construction site. The patient's vital signs were normal

\footnotetext{
Manuscript accepted for publication February 23, 2015

aSaint Joseph's Regional Medical Center, 703 Main Street, Paterson, NJ 07503, USA

bCorresponding Author: Elias Fakhoury, Saint Joseph's Regional Medical Center, 703 Main Street, Paterson, NJ 07503, USA.

Email: Fakhoury.elias@gmail.com
}

doi: http://dx.doi.org/10.14740/jes261w upon arrival, but he was complaining of intense pain over the lower anteromedial aspect of his chest that was associated with the appearance of a bulge in this same area after the fall. Primary and secondary surveys were completed in accordance with the advanced life trauma support (ATLS) protocol and no other life-threatening injuries were found. The patient then underwent computed tomography (CT) of the abdomen and pelvis that demonstrated a large $8 \times 6 \mathrm{~cm}$ left-sided abdominal intercostal hernia with bowel protruding between ribs 10 and 11 (Fig. 1-3). The patient was taken to the operating room for laparoscopic repair of the hernia following informed consent. A Hasson ${ }^{\mathrm{TM}}$ cannula was placed at the umbilicus. The defect between ribs 10 and 11 was visualized and was noted to contain a significant amount of herniated colon and small bowel (Fig. 4, 5). After inspection and confirmation of an intact diaphragm, two additional 5-mm trocars were then placed in the mid-epigastrium and suprapubic regions, respectively. The bowel was reduced into the abdomen and was inspected. A 0.5 $\mathrm{cm}$ serosal tear was noted on the jejunum that was closed with 3-0 silk suture. The hernia defect was then repaired with a flexible composite polypropylene and polyglecaprone- 25 mesh (Ethicon Physiomesh ${ }^{\mathrm{TM}}$ ) in underlay fashion such that there was $3-4 \mathrm{~cm}$ overlap circumscribing the edges of the defect. The mesh was secured in place with transfascial sutures at the

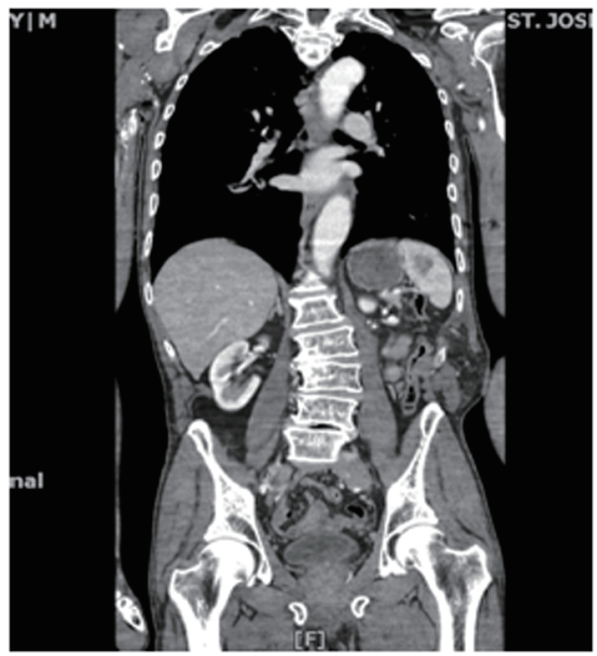

Figure 1. CT coronal view of left intercostal hernia protruding through ribs 10-11. 


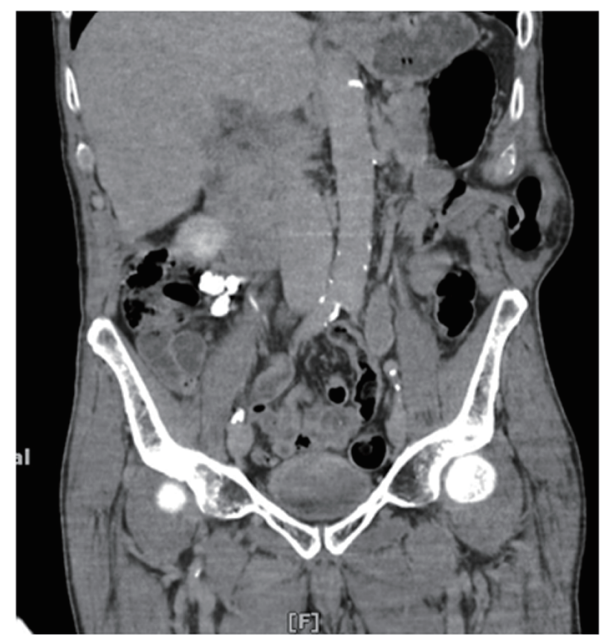

Figure 2. CT coronal view of left intercostal hernia protruding through ribs 10-11.

superior and inferior poles of the mesh and Absorba ${ }^{\mathrm{TM}}$ tacks radially (Fig. 6,7). The patient was discharged home 1 day following surgery with minimal discomfort. The patient was seen subsequently in clinic for his 1-month follow-up without complaint. On physical exam, there appeared to be no sign suggestive of hernia recurrence.

\section{Discussion}

Abdominal hernia repair represents a mainstay of general surgery practice. Abdominal hernias that protrude through the intercostal muscles, however, are a rare phenomenon. Less than 40 cases of transdiaphragmatic intercostal hernias have been reported in the literature $[1,2]$. Even fewer still are cases of intercostal hernias under an intact diaphragm [3]. Intercostal hernias are associated with an increase in abdominal pressure and may develop acutely or over a number of years. A significant or persistent increase in abdominal pressure can tear the intercostal muscles and even fracture underlying ribs. Positive intrathoracic pressure forces the contents out through weak-

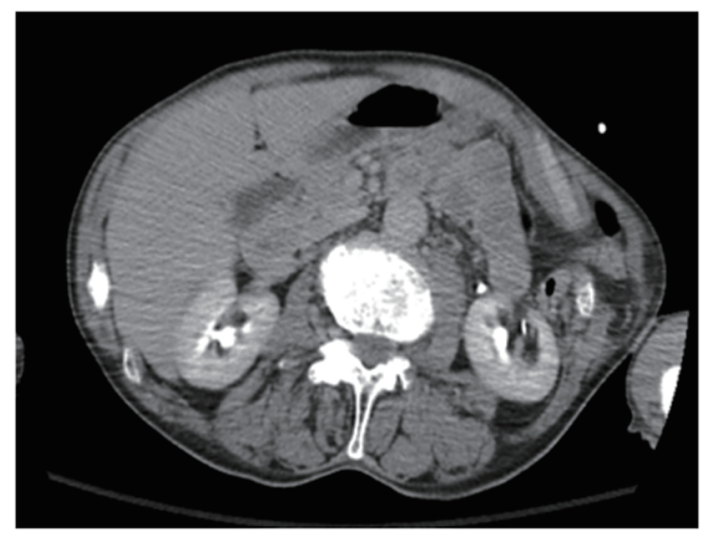

Figure 3. CT cross-section demonstrating left intercostal hernia.

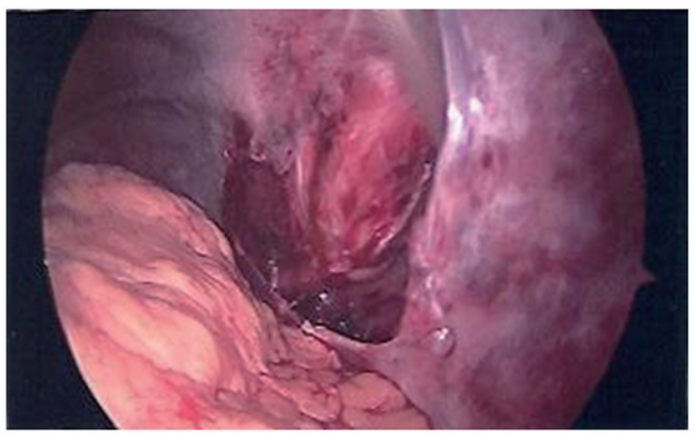

Figure 4. Intraoperative image demonstrating the intercostal hernia between rib 10-11 containing bowel.

ened areas of the chest wall. The majority of cases share the predisposing characteristics of obesity: COPD, advanced age, smokers, and connective tissue disorders [3]. The majority of intercostal hernias are the result of a traumatic mechanism. Sites most susceptible to herniation include: anteriorly at the costochondral junction between the rib and sternum because of lack of external intercostal muscles and posteriorly at the rib vertebral angle due to a lack of internal intercostal muscles $[4,5]$. A careful physical exam in combination with a clinical history will provide the necessary clues to guide clinicians to a correct diagnosis. The typical finding is chest swelling and a reducible bulge on the thoraco-abdominal wall. CT of the chest and abdomen is the gold standard for imaging these hernias, as chest X-ray and ultrasound lack the sensitivity to identify the contents of the hernia and whether there is obstruction or vascular compromise of herniated organs [3]. Surgery is the definitive treatment for intercostal hernias. These hernias should be repaired promptly due to the risk of incarceration. The standard surgical principles of tension free mesh repair should be applied to all abdominal intercostal hernias. One approach to treatment has been to reduce the hernia and close the defect, usually with rib approximation. This open thoracoabdominal approach has been associated with recurrence, although the documented follow-up periods have been short [3]. In stable patients, the intraabdominal contents can be inspected with diagnostic laparoscopy with the possibility of a laparoscopic mesh placement if there is no contraindication to

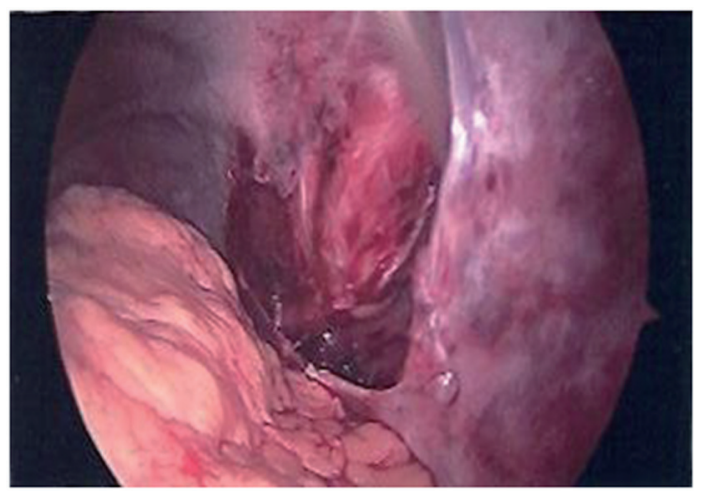

Figure 5. Intraoperative image demonstrating the intercostal hernia completely reduced of all bowel contents. 


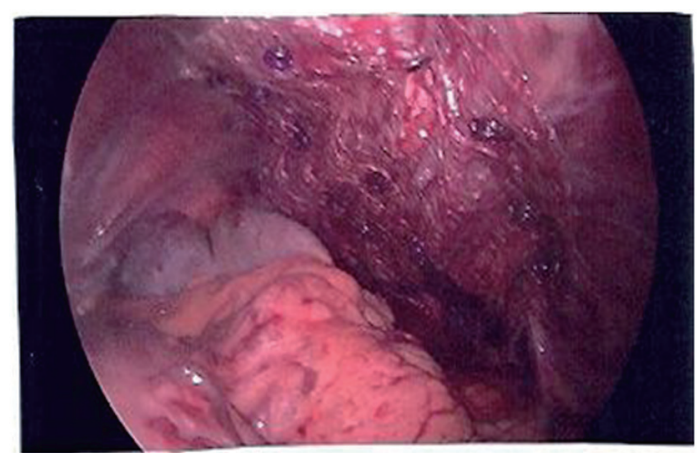

Figure 6. Intraoperative image of laparoscopically repaired intercostal hernia with physiomesh.

do so otherwise. A recently published literature review of abdominal intercostal hernias by Erdas et al described as few as 20 cases of AIH, with only four of these being documented as repaired laparoscopically. Our case demonstrates that a laparoscopic approach to repair intercostal hernias may be feasible in carefully selected patients in the immediate setting of a trauma. The laparoscopic approach is minimally invasive and may serve to blunt the acute-phase response associated with trauma, leading to a faster return to immunological baseline and, in turn, a shorter hospital stay $[6,7]$.

\section{References}

1. Benizri EI, Delotte J, Severac M, Rahili A, Bereder JM, Benchimol D. Post-traumatic transdiaphragmatic intercostal hernia: report of two cases. Surg Today.

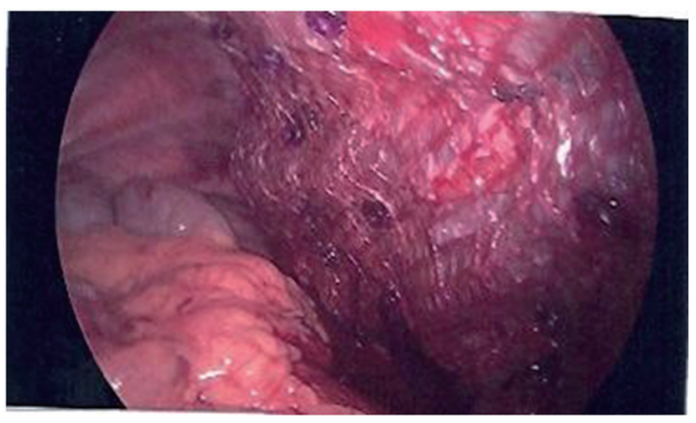

Figure 7. Intraoperative image of laparoscopically repaired intercostal hernia with physiomesh.

2013;43(1):96-99.

2. Sharma OP, Duffy B. Transdiaphragmatic intercostal hernia: review of the world literature and presentation of a case. J Trauma. 2001;50(6):1140-1143.

3. Erdas E, Licheri S, Calo PG, Pomata M. Acquired abdominal intercostal hernia: case report and systematic review of the literature. Hernia. 2014;18(5):607-615.

4. Cole FH, Jr., Miller MP, Jones CV. Transdiaphragmatic intercostal hernia. Ann Thorac Surg. 1986;41(5):565-566.

5. Saw EC, Yokoyama T, Lee BC, Sargent EN. Intercostal pulmonary hernia. Arch Surg. 1976;111(5):548-551.

6. Lennard TW, Shenton BK, Borzotta A, Donnelly PK, White M, Gerrie LM, Proud G, et al. The influence of surgical operations on components of the human immune system. Br J Surg. 1985;72(10):771-776.

7. Buunen M, Gholghesaei M, Veldkamp R, Meijer DW, Bonjer HJ, Bouvy ND. Stress response to laparoscopic surgery: a review. Surg Endosc. 2004;18(7):1022-1028. 